\title{
Single cell eQTL analysis identifies cell type-specific genetic control of gene expression in fibroblasts and reprogrammed induced pluripotent stem cells
}

Drew Neavin ${ }^{1 \dagger}$, Quan Nguyen ${ }^{2 \dagger}$, Maciej S. Daniszewski ${ }^{3,4,5}$, Helena H. Liang ${ }^{3,4}$, Han Sheng Chiu², Yong Kiat Wee ${ }^{1}$, Anne Senabouth', Samuel W. Lukowski², Duncan E. Crombie ${ }^{3,4}$, Grace E. Lidgerwood ${ }^{3,4,5}$, Damián Hernández ${ }^{3,4,5}$, James C. Vickers ${ }^{6}$, Anthony L. Cook ${ }^{6}$, Nathan J. Palpant ${ }^{2 \dagger}$, Alice Pébay ${ }^{3,4,5+}$, Alex W. Hewitt ${ }^{3,4,7 \dagger}$ and Joseph E. Powell ${ }^{1,8^{*}+}$

* Correspondence: j.powell@garvan. org.au

${ }^{\dagger}$ Nathan J. Palpant, Alice Pébay, Alex W. Hewitt and Joseph E. Powell are joint senior authors.

${ }^{\dagger}$ Drew Neavin and Quan Nguyen are co-first authors.

${ }^{1}$ Garvan-Weizmann Centre for Cellular Genomics, Garvan Institute of Medical Research, Darlinghurst, Sydney, Australia

${ }^{8}$ UNSW Cellular Genomics Futures Institute, School of Medical

Sciences, University of New South Wales, Sydney, Australia

Full list of author information is available at the end of the article

\begin{abstract}
Background: The discovery that somatic cells can be reprogrammed to induced pluripotent stem cells (iPSCs) has provided a foundation for in vitro human disease modelling, drug development and population genetics studies. Gene expression plays a critical role in complex disease risk and therapeutic response. However, while the genetic background of reprogrammed cell lines has been shown to strongly influence gene expression, the effect has not been evaluated at the level of individual cells which would provide significant resolution. By integrating single cell RNA-sequencing (scRNA-seq) and population genetics, we apply a framework in which to evaluate cell type-specific effects of genetic variation on gene expression.
\end{abstract}

Results: Here, we perform scRNA-seq on 64,018 fibroblasts from 79 donors and map expression quantitative trait loci (eQTLs) at the level of individual cell types. We demonstrate that the majority of eQTLs detected in fibroblasts are specific to an individual cell subtype. To address if the allelic effects on gene expression are maintained following cell reprogramming, we generate scRNA-seq data in 19,967 iPSCs from 31 reprogramed donor lines. We again identify highly cell type-specific eQTLs in IPSCs and show that the eQTLs in fibroblasts almost entirely disappear during reprogramming.

Conclusions: This work provides an atlas of how genetic variation influences gene expression across cell subtypes and provides evidence for patterns of genetic architecture that lead to cell type-specific eQTL effects.

Keywords: Expression quantitative trait loci (eQTLs), Single cell RNA-sequencing (scRNA-seq), Induced pluripotent stem cells (iPSCs) 


\section{Background}

Mapping expression quantitative trait loci (eQTLs) is a powerful method to study how common genetic variation between individuals influences gene expression [1, 2]. To date, nearly all eQTL studies have been conducted while interrogating 'bulk' samples, where the RNA is collected from millions of lysed cells, and therefore, gene expression represents an average across all cells in a sample. However, even 'bulk' eQTL studies in different tissues $[3,4]$ and cultured cell lines $[5,6]$ have revealed specificity in both the presence and allelic effects of eQTLs [7, 8]. Single cell approaches have already revealed that stem cell cultures do not contain a single homogeneous cell type $[5,6,9]$, but instead consist of multiple cell types that have unique transcriptional profiles. For this study, we harnessed recent technological advances for high-throughput generation of single cell data that leveraged cell multiplexing from multiple donors [10-12]. This experimental framework enabled the identification of cell type-specific genetic effects on gene expression which revealed eQTLs that were cell type specific and that would not be detected by 'bulk' approaches.

Previous studies have identified cell type-specific eQTLs using scRNA-seq which were unobservable in bulk RNA-sequence studies [13-17]. The first study to report this enhanced cell type-specific eQTL detection from scRNA-seq investigated 92 genes measured in 1440 single cells from lymphoblastoid cell lines in 15 individuals [15]. In the current study, we set out to understand the impact of common genetic variants on gene expression in fibroblast and reprogrammed iPSC cell types through eQTL mapping at the level of cell subpopulations.

\section{Results}

To identify cell type-specific eQTLs in an unbiased manner, we generated scRNA-seq expression profiles of 83,985 cells-64,018 cultured dermal fibroblasts, generated from skin biopsies from 79 unrelated individuals, and 19,967 iPSCs reprogrammed from 31 of the dermal fibroblast lines (Fig. 1a). After quality control, we used an unsupervised clustering approach [18] to identify six types of fibroblasts and four types of iPSCs (Fig. 1b, c). Fibroblast and iPSC types contained equal distributions of individual donors, pool batches and cell cycle states (Additional file 1: Figure S1 and S2). Cell types were classified based on the relative activity of the regulating transcription factors in fibroblasts $\left(\mathrm{SIX}^{+}, \mathrm{HOXC6}^{+}, \mathrm{ATF}^{+}, \mathrm{TEAD}^{+}, \mathrm{KLF}^{+} 0^{+}\right.$and $\left.\mathrm{RXRB}^{+}\right)$and $\mathrm{iPSCs}\left(\mathrm{HIC}^{+}\right.$, $\mathrm{ATF}^{+}, \mathrm{BRF}^{+}$and $\mathrm{CEBPG}^{+}$) (Fig. 1d, e; Additional file 2: Table S1 and Additional file 3: Table S2; and Table 1). Further, pseudo-trajectory analysis demonstrated that the identified cell types were positioned along a clear lineage trajectory for both fibroblast and iPSC types which was exemplified by the top differentially expressed genes (Additional file 1: Figure S3-4, Additional file 4: Table S3 and Additional file 5: Table S4). We also used an unbiased approach to classify cells against reference transcriptome profiles from the human primary cell atlas $[19,20]$, which demonstrated that the majority of fibroblasts mapped to the fibroblast or mesenchymal stem cell (MSC) reference, while the majority of iPSCs mapped to the iPSC or embryonic stem cell references (Additional file 1: Figure S5A-B). Due to the phenotypic and transcriptional similarities of fibroblasts and MSCs (Additional file 1: Figure S5C), it is not surprising that some fibroblast cells mapped to the MSC reference [21]. 


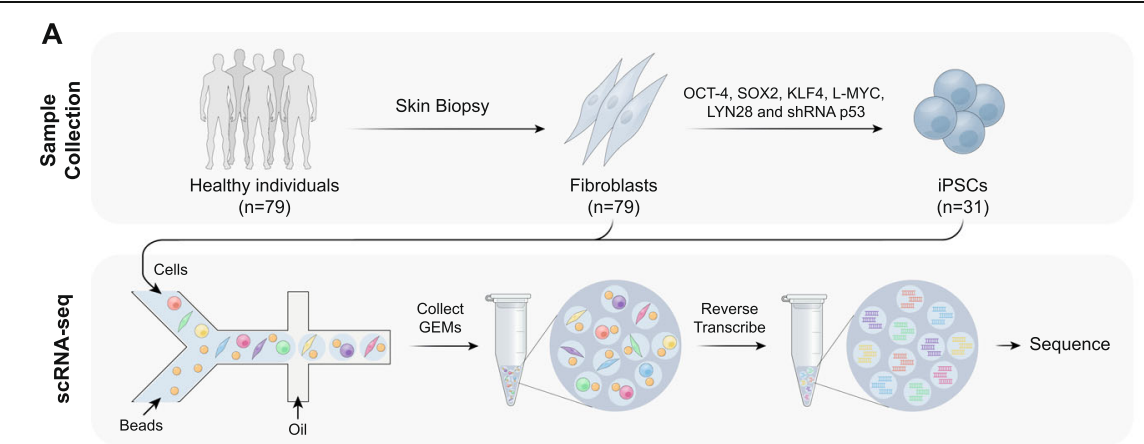

B

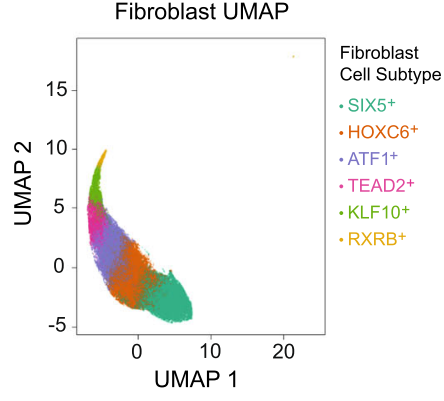

D

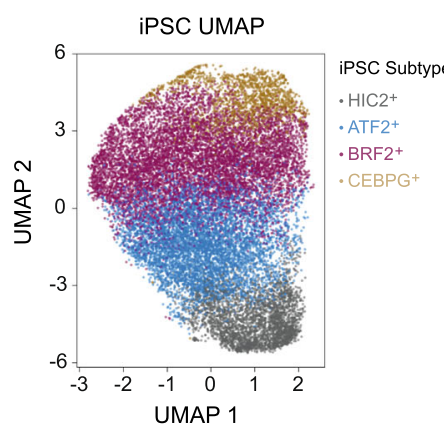

C

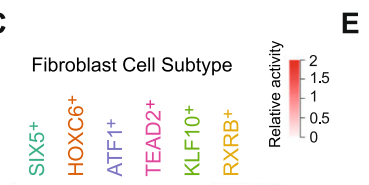

$E$

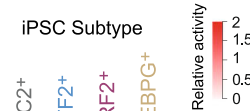

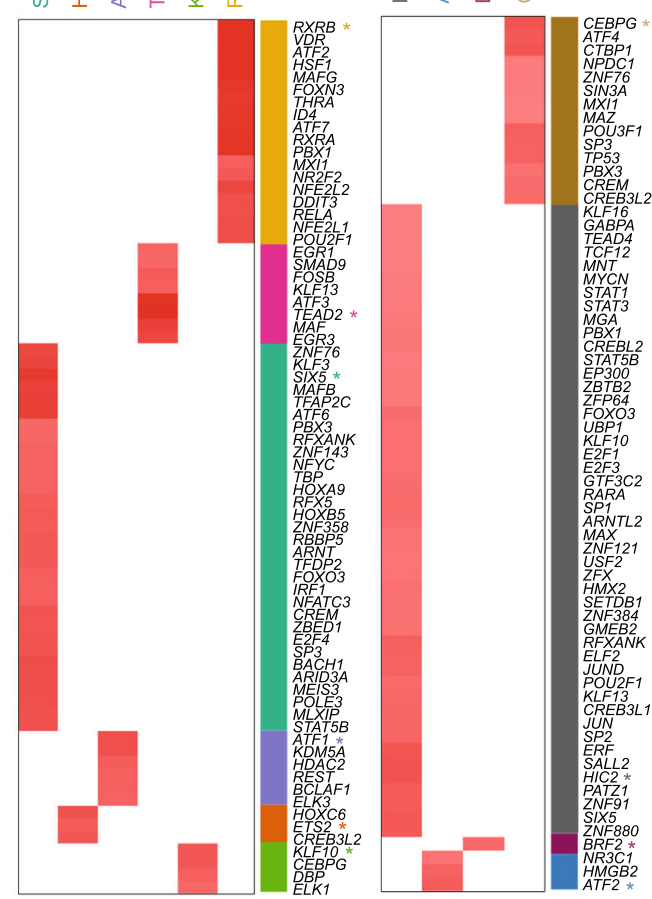

Fig. 1 Fibroblast and iPSC cluster characterisation. a This study used skin biopsies to generate fibroblasts from 79 healthy volunteers and reprogrammed them into induced pluripotent stem cell (iPSC) lines for 31 of the original 79 individuals. b Six fibroblast subtypes were identified from the transcriptional profiles of 64,018 single fibroblast cells. c The 50 TFs with the highest relative activities as identified by SCENIC in the six fibroblast subtypes. d Four iPSC subtypes were identified from 19,967 single iPSCs. e The 50 TFs with the highest relative activities as identified by SCENIC in the four iPSC subtypes. Asterisk indicates the genes used to name each subtype

We subsequently tested for cis-eQTLs independently in each of the 10 cell types. We identified a total of 46,103 eQTLs for 2958 genes (FDR $<0.1$ ) across all cell types-45, 503 eQTLs for 2887 genes in fibroblast cell types and 810 cis-eQTLs for 86 genes in iPSC cell types (Additional file 7: Table S6 and Additional file 8: Table S7, Table 1). The majority of cis-eQTLs were predominantly cell type specific, with $77.6 \%$ of the eGenes-genes that have an eQTL-(71.5\% of the cis-eQTLs) identified in only one fibroblast type (Fig. 2a, b and Additional file 1: Figure S6A). However, neighbouring fibroblast types were more likely to share common eGenes than distant fibroblast types 
Table 1 Summary of fibroblast type cis-eQTL. The median number of cells per individual, the number of significant eSNPs detected, the number of significant eGenes detected and the number of unique eGenes per cell type are enumerated

\begin{tabular}{lllll}
\hline Cell type & $\begin{array}{l}\text { Median cells } \\
\text { per individual }\end{array}$ & $\begin{array}{l}\text { Number of significant } \\
\text { eSNP-eGene pairs }\end{array}$ & $\begin{array}{l}\text { Number of } \\
\text { significant eGenes }\end{array}$ & $\begin{array}{l}\text { Unique genes } \\
\text { with cis-eQTL }\end{array}$ \\
\hline Fibroblast SIX5+ & 282 & 13,886 & 664 & 383 \\
Fibroblast HOXC6+ & 253 & 23,348 & 1030 & 615 \\
Fibroblast ATF1+ & 175 & 20,105 & 916 & 500 \\
Fibroblast TEAD2+ & 46 & 6351 & 402 & 232 \\
Fibroblast KLF10+ & 36 & 4567 & 299 & 163 \\
Fibroblast RXRB+ & 7 & 2883 & 517 & 339 \\
iPSC HIC2+ & 240 & 52 & 6 & 5 \\
iPSC ATF2+ & 176 & 14 & 3 & 2 \\
iPSC BRF2+ & 74 & 52 & 11 & 7 \\
iPSC CEBPG & 41 & 695 & 68 & 55 \\
\hline
\end{tabular}

(Fig. 2b, c). iPSC types also demonstrated a high percentage of cell type-specific eQTLs with $97.2 \%$ of the eGenes (99.6\% of the cis-eQTLs) identified in only one iPSC type (Additional file 1: Figure S6B-C). Since each cell type was characterised by activity of a specific set of transcription factors (Fig. 1c, e), we tested whether any of the eQTLs were predicted to alter transcription factor binding in those cell types and identified multiple loci that were predicted to alter key transcription factor binding (Additional file 8: Table S7).

Cell type ubiquitous eQTLs (shared across all fibroblasts or iPSC cell types) were rare, with seven eGenes in fibroblasts (Fig. 2a, Additional file 1: Figure S6A and S7) and none in iPSCs (Additional file 1: Figure S6B). Looking across the cell reprogramming event, we observed a complete lack of shared eQTLs between fibroblasts and iPSCs. Only 14 genes had eQTLs in both fibroblasts and iPSCs (Fig. 2d), but none of those shared a common eSNP-a SNP significantly associated with an eGene-or eSNPs in linkage disequilibrium with one another $\left(r^{2}<0.2\right)$, which indicates that their expression is likely associated with independent loci (Fig. $2 \mathrm{~d}$ and Additional file 1: Figure S8). These cell type-specific eQTLs are clearly exemplified by top eQTLs identified for each of the 10 cell types (Fig. 2e).

We then investigated whether the eQTLs identified in fibroblasts replicated in bulk RNA-sequence data from the Genotype-Tissue Expression database (GTEx, cultured fibroblasts $n=483$ ) [22]. Only $41.1 \%$ of the 45,503 eQTLs identified in the six fibroblast types replicated in GTEx, although they demonstrated a consistent shared direction of allelic effects. One explanation for this observation is that bulk RNA-seq approaches mask cell type-specific effects through averaged gene expression across cells. Therefore, we hypothesised that cell type ubiquitous eQTLs (from the single cell analysis) would have higher replication rates compared to cell typespecific eQTLs. eQTLs that were shared across multiple scRNA-seq fibroblast cell types showed a highly significant difference compared with eQTLs that were significant in just one fibroblast type $\left(p<5 \times 10^{-324}\right.$ for eGenes and $p=6 \times 10^{-150}$ for eSNPs; Fig. 2a and Additional file 1: Figure S6A). Further, we identified that the allelic effect size of the eGenes and eQTLs in GTEx cultured fibroblasts was positively correlated with the number of fibroblast types where those eGenes and 


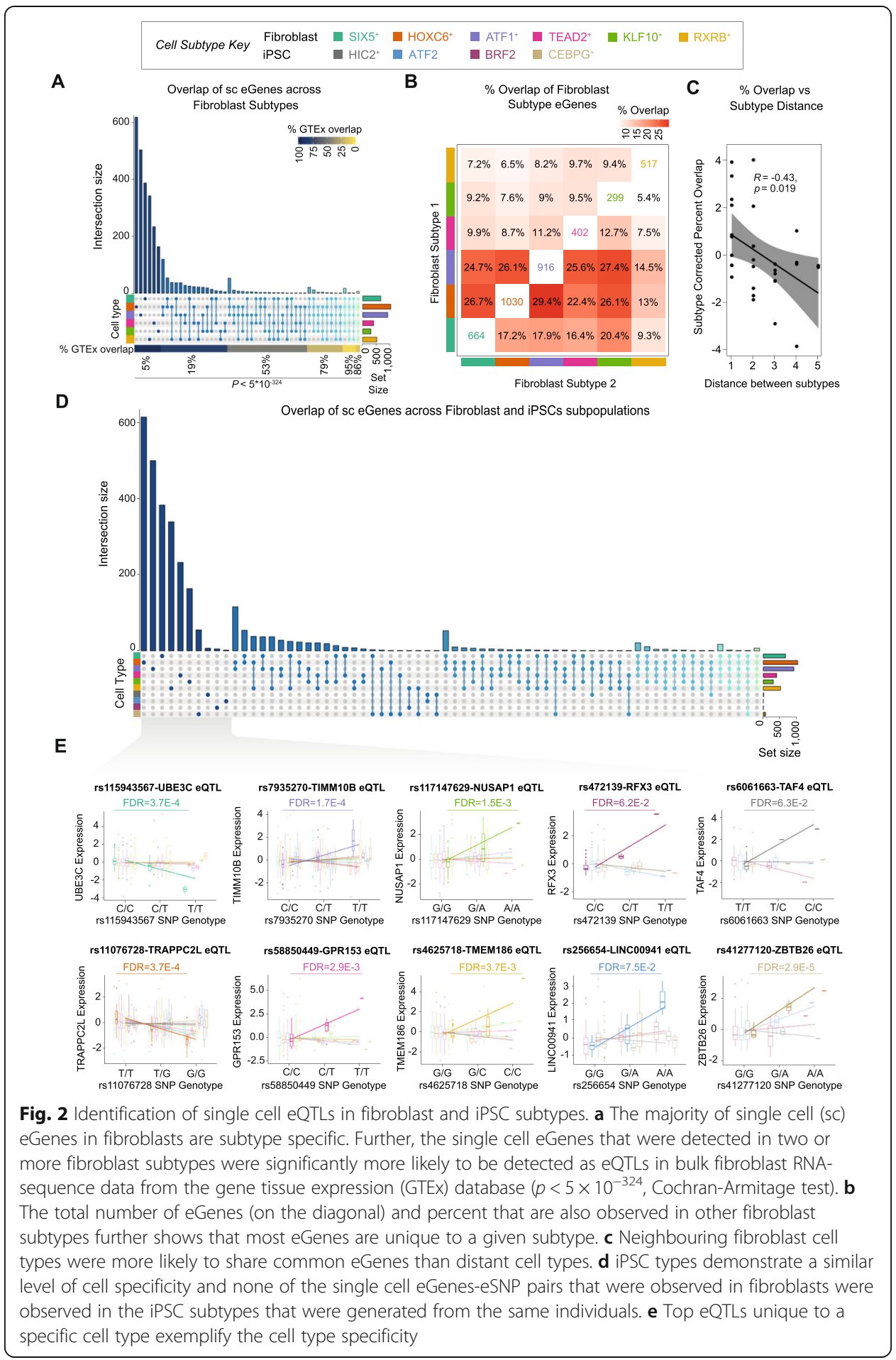

eQTL were significant (Additional file 1: Figure S9). These results indicate that eQTL mapping using bulk RNA-sequence data is likely not sensitive enough to identify fibroblast type-specific eQTLs.

Based on our initial observation that cell type eQTL effects are highly specific, we next sought to identify how different types of genetic architecture and gene expression patterns contributed to cell type-specific effects in fibroblasts and iPSCs. 
One potential explanation for the cell type-specific eQTL detection is that the gene is only expressed in one cell type, and therefore, we would not expect to observe an eQTL in a cell type that does not express the gene. To evaluate this possibility, we correlated the expression of each eGene, with its expression levels in each of the other cell types (Additional file 1: Figure S10). Gene expression was highly correlated, which indicates that cell type-specific eQTL is not a function of cell type-specific gene expression. Another possible explanation for the cell typespecific eQTLs is low statistical power to detect eQTLs in multiple cell types. We tested this hypothesis in two ways: (1) we correlated the eQTL effect sizes between different cell types and (2) we implemented an empirical framework to test for enrichment of cell type-specific eGenes in other cell types. The cell type-specific eQTL allelic effects (betas) were not well correlated in other cell types unless they were already identified as significant in the other cell type (Additional file 1: Figure S11). However, there was some enrichment of the test statistic across cell typesmostly for fibroblast types that were similar to one another such as $\operatorname{SIX~}^{+}$and $\mathrm{HOXC6}^{+}$(Additional file 1: Figure S12). While larger studies will be required to fully elucidate the full degree of cell type specificity, these results suggest that many of the eQTLs identified are cell type specific. Therefore, we conclude that the majority of cell type-specific eQTLs that we have identified were not a result of differences in gene expression or due to lack of statistical power. We next set out to interrogate eGenes that were in common between multiple cell types.

We identified 283 eGenes that were significant in multiple cell types, but which had different top eSNPs-255 eGenes in at least two fibroblast types, no eGenes with different top eSNPs in iPSC types and 11 eGenes with different top eSNPs in a fibroblast type and an iPSC type. In these instances, we considered two alternative hypotheses: (1) that there was one eQTL shared between cell types but that it was tagged by a different top eSNP in each cell type, or (2) that there were two independent cell typespecific eQTLs for the same gene. To address these hypotheses, we tested whether the top eSNP in a given cell type was still significantly associated with gene expression after correcting for the top eSNP in the other cell type-the same method used for conditional eQTL analyses [23]. A significant association of the SNP with the eGene expression after correction for the other eSNP would indicate that the two eSNPs were not tagging the same eQTL and were, therefore, independent loci. The analysis identified that between 28.6 and $55.7 \%$ of these loci for a given fibroblast type were independent (Fig. 3 and Additional file 9: Table S8), and 100\% of the eGenes shared between the fibroblast and iPSC types were also independent loci (Additional file 9: Table S8). These results denote that many of the eGenes that were shared between multiple cell types are in fact regulated by different loci, providing further support to our previous finding that the majority of eQTLs are cell type specific.

Indeed, we identified that even though the Kelch Like Family Member 36 (KLHL36) gene was a significant eGene in three fibroblast types, it was regulated by independent loci in each cell type (Fig. 4a-d). Further, the top eSNPs for each locus were not in LD with one another $\left(r^{2}<0.1\right)$ (Fig. 4e). KLHL36 is highly expressed in fibroblasts compared to other cell types in the Genotype-Tissue Expression (GTEx) database (Additional file 1: Figure S13) suggesting that it may play an important role in fibroblast biology. Further, KLHL36 is part of the E3 ubiquitin ligase family which has been 


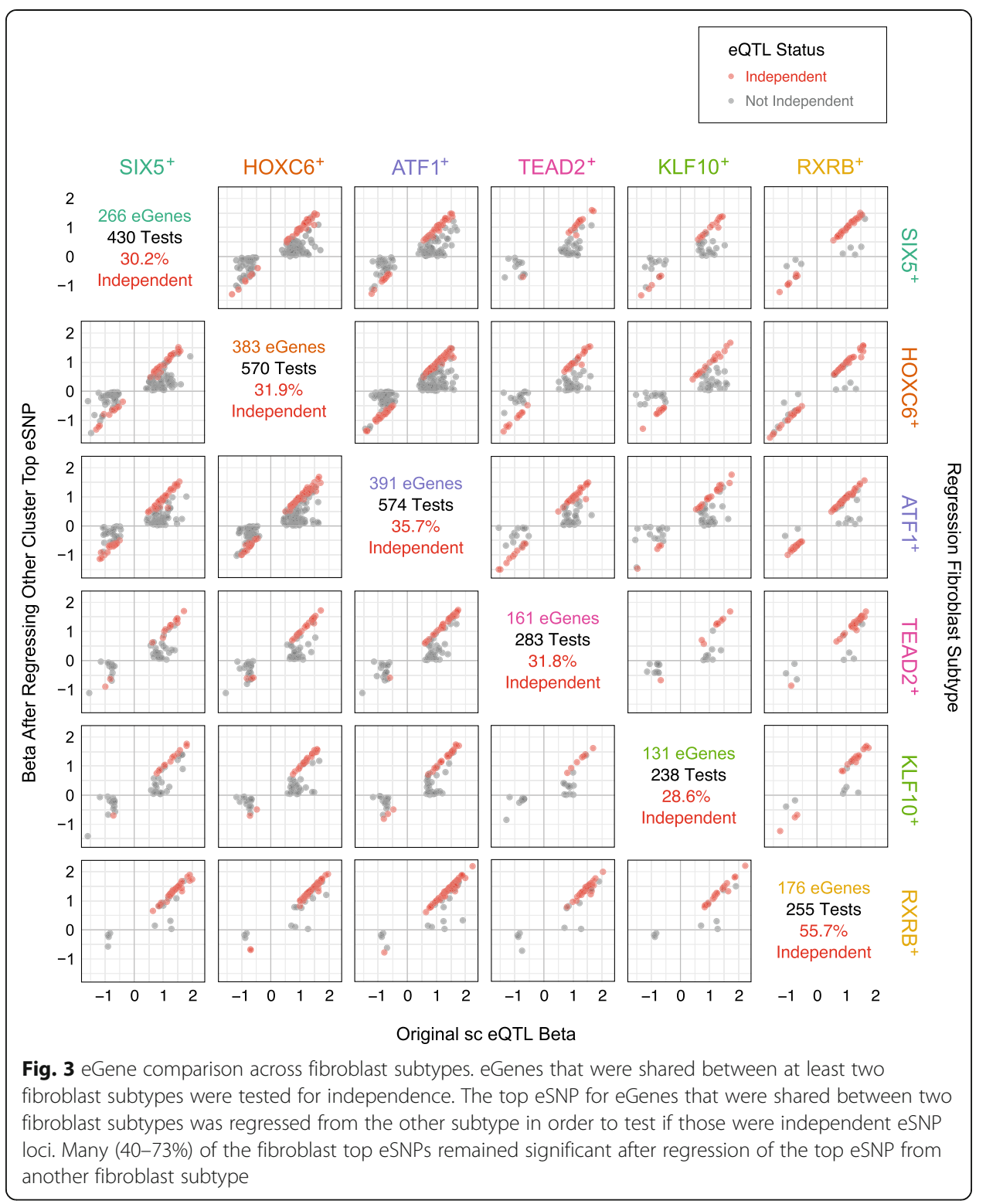

implicated in skin fragility [24] and fibroblast pseudopodia function [25]-again highlighting the potential role of this gene in fibroblast biology and physiology.

Next, we investigated the 153 eGenes that shared at least one significant eSNP-eGene pair across multiple cell types. We evaluated the potential interactions between cell type and eSNP that would lead to a difference in magnitude of the allelic effect in different cell types. In cases where multiple eSNP-eGene pairs were significant for the same eGene across multiple cell types, we tested the eSNP-eGene pair with the largest beta difference between two fibroblast types. This analysis identified 42 (21.2\%) significant eSNP-fibroblast type interactions at an FDR of 0.05 (Additional file 10: Table S9). We identified a number of ubiquitous eQTLs, whose allelic effects were significantly different across cell types.

Next, we asked whether any of the eQTLs that we identified might impact fibroblast reprogramming to iPSCs. We first asked whether there were eQTLs for 


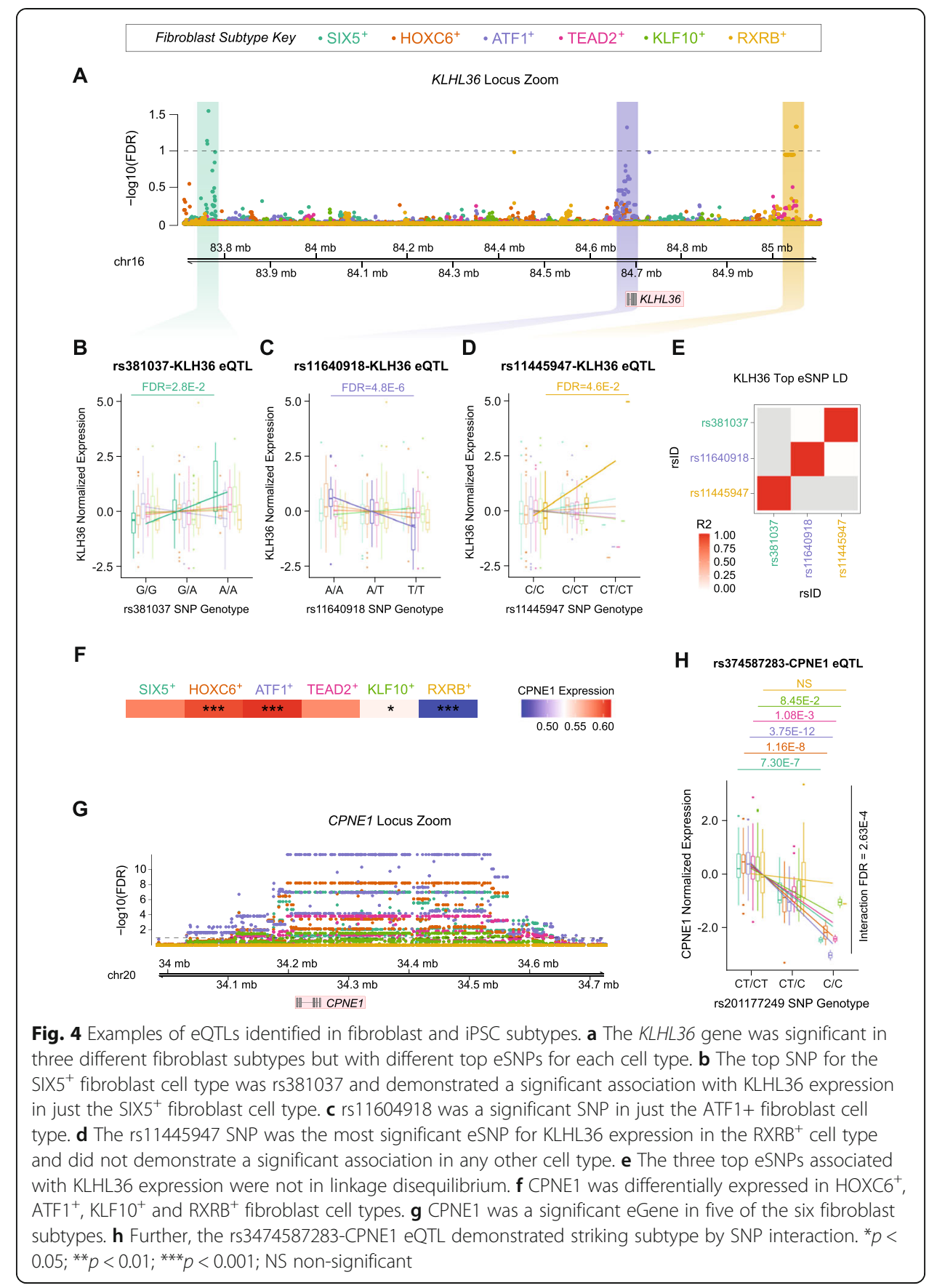

any genes that characterise cell types as they transition from fibroblasts to iPSCs. We identified multiple eQTLs for genes that characterised cell types during the transition from fibroblasts to iPSCs (Additional file 1: Figure S14A). In addition, $76.5 \%$ of those reprogramming eQTLs were unique to one fibroblast subtype which suggests that these genetic variants may impact reprogramming efficiency to varying degrees depending on the cell type. Further, we tested for eQTLs in regions differentially methylated post reprogramming (Additional file 1: Figure S14B) and in regions that are incompletely reprogrammed in some iPSCs relative to embryonic stem cells (ESCs) (Additional file 1: Figure S14C). In both cases, we 
identified multiple eQTLs, the majority of which were cell type specific-68.6\% in regions differentially methylated between fibroblasts and iPSCs and $62.1 \%$ in regions differentially methylated between iPSCs and ESCs, indicating that these genetic variants may influence iPSC reprogramming. Of interest, this analysis identified genes that have previously been associated with reprogramming efficiency such as DNAJ15 [26] or previously been reported as eQTLs such as MICA [27] and CPNE1 [28].

In addition, CPNE1 was differentially expressed between cell types in our dataset (Additional file 1: Figure S4F and Additional file 4: Table S3) and the rs374587283CPNE1 cis-eQTL demonstrated a significant SNP-cell type interaction ( $p=$ $2.64 .9 \times 10^{-04}$; Fig. 4g, h). CPNE1 is a calcium-dependent binding protein that is thought to play an important role in membrane trafficking, and is important for neuronal cell differentiation [29,30]. Therefore, this eQTL could impact the differentiation potential of iPSC lines toward neuronal lineages.

\section{Discussion}

We set out to identify and define the dynamics of eQTLs in fibroblasts and fibroblastderived iPSC types. Collectively, our results provide evidence that there is a high degree of cell type-specific gene regulation that is not captured with bulk RNA-seq. Our results indicate that even when the same eGene is observed in different cell types, the allelic effect may be altered in different cell types or may be regulated by different loci entirely. Our findings support previous reports that many cell type-specific eQTLs are not detected using bulk RNA-sequencing and that scRNA-seq can be utilised to enhance eQTL detection [31].

These results contrast slightly with GTEx [22] that showed that multiple eQTLs are common across tissues. However, it is important to note that in GTEx, the RNA expression levels are the averaged signal from all cell types in the tissue biopsy. While the cellular composition of tissues differs, most tissues share common cell types such as epithelial cells, circulating immune cells and adipocytes. Therefore, under a scenario of highly cell type-specific eQTLs, we would expect to see shared eQTLs between tissues due to the common cell types that are shared between multiple tissues. Likewise, truly cell type ubiquitous eQTLs would replicate at a higher frequency between single cell eQTLs and GTEx - as we observe here (Fig. 2). Nevertheless, the results we present here strongly support the hypothesis of true cell type-specific eQTLs as we observe little correlation of the allelic effects (betas) between cell types. However, to adequately uncover the relationship between bulk and single cell eQTLs, it is likely that future studies which generate both types of data will be required.

In addition, our analyses identified novel cis-eQTLs for genes that characterise cell types during iPSC reprogramming [32], located in functional genomic regions that reflect reprogramming efficiency based on epigenetic profiles [33, 34]. Those loci also demonstrated important cell type specificity that could impact iPSC reprogramming and pluripotency in a cell type-specific manner. However, additional studies will be required to fully understand the mechanisms behind how these genetic loci might impact iPSC reprogramming in lines that are derived from different individuals. Specifically, CellTag $[35,36]$ or other barcoding methods will enable cells to be both collected and tracked throughout the entire reprogramming event or differentiation lineage. 
scRNA-seq provides a number of advantages over bulk RNA-sequencing for eQTL mapping. Specifically, scRNA-seq enables cell types to be identified in an unbiased manner before eQTL detection. Therefore, even cell types that have previously not been described or well characterised can be identified and separated for eQTL mapping, thereby decreasing the measurement noise that is introduced due to heterogeneity of cells in bulk RNA-sequence profiling. Furthermore, scRNA-seq enables the cells from multiple individuals to be pooled in a single experiment, thereby decreasing technical batch effects that can confound biological variation between individuals.

Larger studies will be required to fully parse out the cell type-specific effects.

\section{Conclusions}

This study has provided a map of eQTLs in fibroblast and fibroblast-derived iPSC types that will be an important reference for future studies in iPSC-derived cell types.

\section{Methods}

\section{Participant recruitment and ethics approval}

Experimental work was approved by the Human Research Ethics committees of the Royal Victorian Eye and Ear Hospital (11/1031), University of Melbourne (1545394) and University of Tasmania (H0014124) in accordance with the requirements of the National Health \& Medical Research Council of Australia (NHMRC) and conformed with the Declaration of Helsinki [37].

\section{Fibroblast culture}

Human skin punch biopsies were obtained from subjects over the age of 18 years. Fibroblasts were cultured in DMEM high glucose supplemented with $10 \%$ foetal bovine serum (FBS), L-glutamine, penicillin $(100 \mathrm{U} / \mathrm{mL})$ and streptomycin $100(\mu \mathrm{g} / \mathrm{mL})$ (all from Thermo Fisher Scientific, USA). All cell lines were mycoplasma-free (MycoAlert mycoplasma detection kit, Lonza, Switzerland).

\section{Generation and maintenance of iPSCs}

Human iPSCs were reprogrammed from fibroblast cultures by nucleofection (Amaxa ${ }^{\text {тx }}$ Nucleofector $^{\mathrm{mw}}$ ) of episomal vectors expressing OCT-4, SOX2, KLF4, L-MYC, LIN28 and shRNA against p53 [38], in feeder- and serum-free conditions using $\mathrm{TeSR}^{\mathrm{mm}}-\mathrm{E}^{\mathrm{im}}$ medium (STEMCELL Technologies, Canada) and selected by sorting with anti-human TRA-1-60 Microbeads using a MultiMACS (Miltenyi, Germany) as described in [39] and [40]. Cells were maintained on vitronectin $\mathrm{XF}^{\mathrm{m}}$ (STEMCELL Technologies)-coated plates using $\mathrm{TeSR}^{\mathrm{mix}}-\mathrm{E}^{\mathrm{mi}}$ (Stem Cell Technologies). At passage eight, cells were assessed for quality control as described previously [40].

\section{iPSC quality control}

Pluripotency was assessed by immunochemistry for expression of OCT3/4 (sc-5279, Santa Cruz Biotechnology, USA) and TRA-1-60 (MA1-023-PE, Thermo Fisher Scientific). Copy number variation (CNV) analysis of original fibroblasts and iPSCs was performed using Illumina HumanCore Beadchip arrays with PennCNV [41, 42] and QuantiSNP [42] with default parameter settings. Chromosomal aberrations were 
deemed to involve $\geq 20$ contiguous SNPs or a genomic region spanning $\geq 1 \mathrm{MB}$ [41, $42]$. The $B$ allele frequency (BAF) and the log $\mathrm{R}$ ratio (LRR) were extracted from GenomeStudio (Illumina, USA) for representation.

\section{Generating the single cell RNA-sequence data}

Viable cells were sorted on a BD Influx cell sorter (Becton-Dickinson) using propidium iodide into Dulbecco's phosphate-buffered saline (PBS) $+0.1 \%$ bovine serum albumin and retained on ice. Sorted cells were counted and assessed for viability with Trypan Blue using a Countess automated counter (Invitrogen) and then resuspended at a concentration of $800-1000$ cells $/ \mu \mathrm{L}\left(8 \times 10^{5}\right.$ to $1 \times 10^{6}$ cells $\left./ \mathrm{mL}\right)$. Final cell viability estimates ranged between 92 and $96 \%$.

Single cell suspensions were loaded onto 10X Genomics Single Cell 3' Chips along with the reverse transcription (RT) mastermix as per the manufacturer's protocol for the Chromium Single Cell 3' Library (10X Genomics; PN-120233), to generate single cell gel beads in emulsion (GEMs). Reverse transcription was performed using a C1000 Touch Thermal Cycler with a Deep Well Reaction Module (Bio-Rad) as follows: $55^{\circ} \mathrm{C}$ for $2 \mathrm{~h}, 85^{\circ} \mathrm{C}$ for $5 \mathrm{~min}$, hold $4{ }^{\circ} \mathrm{C}$. cDNA was recovered and purified with DynaBeads MyOne Silane Beads (Thermo Fisher Scientific; Cat\# 37002D) and SPRIselect beads (Beckman Coulter; Cat\# B23318). Purified cDNA was amplified as follows: $98^{\circ} \mathrm{C}$ for 3 min; $12 \mathrm{x}\left(98^{\circ} \mathrm{C}\right.$ for $15 \mathrm{~s}, 67^{\circ} \mathrm{C}$ for $20 \mathrm{~s}, 72^{\circ} \mathrm{C}$ for $\left.60 \mathrm{~s}\right) ; 72^{\circ} \mathrm{C}$ for $60 \mathrm{~s}$; hold $4{ }^{\circ} \mathrm{C}$. Amplified cDNA was purified using SPRIselect beads and sheared to approximately $200 \mathrm{bp}$ with a Covaris S2 instrument (Covaris) using the manufacturer's recommended parameters. Sequencing libraries were generated with unique sample indices (SI) for each chromium reaction. Libraries were multiplexed and sequenced on an Illumina NextSeq 500 (NextSeq control software v2.0.2/Real Time Analysis v2.4.11) using a 150-cycle NextSeq 500/550 High Output Reagent Kit v2 (Illumina, FC-404-2002) in standalone mode as follows: 98 bp (Read 1), 14 bp (I7 Index), 8 bp (I5 Index) and 10 bp (Read 2).

\section{scRNA-seq Cellranger processing}

Processing of the sequencing data into transcript count tables was performed using the Cell Ranger Single Cell Software Suite by 10X Genomics [43]. Raw base call files from the NextSeq 500 sequencer were demultiplexed, using the cellranger mkfastq pipeline, into sample-specific FASTQ files. These FASTQ files were then processed with the cellranger count pipeline where each sample was processed independently. First, cellranger count used STAR to align cDNA reads to the hg19 human reference transcriptome, which accompanied the Cell Ranger Single Cell Software Suite [44]. We note that, since the expression data is limited to the $3^{\prime}$ end of a gene and we used gene-level annotations, differences between reference versions, such as GRCh38, are unlikely to significantly alter conclusions. Aligned reads were filtered for valid cell barcodes and unique molecular identifiers (UMIs) and observed cell barcodes were retained if they were 1-Hamming-distance away from an entry in a whitelist of known barcodes. UMIs were retained if they were not homopolymers and had a quality score $>10$ (90\% base accuracy). Cellranger count corrected mismatched barcodes if the base mismatch was due to sequencing error, determined by the quality of the mismatched base pair and the overall distribution of barcode counts. A UMI was corrected to another, more 
prolific UMI if it was 1-Hamming-distance away and it shared the same cell barcode and gene. Cellranger count examined the distribution of UMI counts for each unique cell barcode in the sample and selected cell barcodes with UMI counts that fell within the 99th percentile of the range defined by the estimated cell count value. The default estimated cell count value of 3000 was used for this experiment. Counts that fell within an order of magnitude of the 99th percentile were also retained. The resulting analysis files for each sample were then aggregated using the cellranger aggr pipeline, which performed a between-sample normalisation step and merged all samples into one. Post-aggregation, the count data was processed and analysed using a comprehensive pipeline assembled and optimised in-house as described below.

To pre-process the mapped data, we constructed a cell quality matrix based on the following data types: library size (total mapped reads), the total number of genes detected, percent of reads mapped to mitochondrial genes and percent of reads mapped to ribosomal genes (Additional file 1: Figure S15). Cells that had any of the four parameter measurements that were greater than $3 x$ median absolute deviation (MAD) of all cells were considered outliers and removed from subsequent analysis. In addition, we applied two thresholds to remove cells with mitochondrial reads above $20 \%$ or ribosomal reads above $50 \%$. To exclude genes that were potentially detected from random noise, we removed genes that were detected in fewer than $1 \%$ of all cells. These quality control filters resulted in consistent total reads per individual and per pool in both fibroblasts and iPSCs (Additional file 1: Figure S16). Before normalisation, abundantly expressed ribosomal genes and mitochondrial genes were discarded to minimise the influence of those genes in driving clustering and differential expression analysis.

\section{Demultiplexing}

We adapted the Demuxlet method to our 10x scRNA-seq data [16]. The likelihood that a cell originated from a sample is the cumulative likelihood of single nucleotide polymorphism genotypes identified in each cell. We calculated posterior probability of a genotype $g$ identified for a cell based on scRNA-seq data given the DNA data from the imputed BeadChip genotypes. Since the single cell SNP genotype data is sparse, to increase the coverage of SNPs called from scRNA-seq data that are in the SNP genotype data, we imputed SNP genotypes using the haplotype reference panel. We applied an ensemble approach using the outputs from pre-imputed genotype data, imputed genotype likelihood data, and impute genotype dosage data, increased the singlet probabilities from Demuxlet (Additional file 1: Figure S17). The ensemble approach enabled the unique donor assignment of $90.6 \%$ of all cells, with high confidence to each sample, where Demuxlet predicted no ambiguously assigned droplets. Of note, $100 \%$ of the cells before Demuxlet were identified in the cellranger pipeline as a singlet. Demuxlet identified $90.6 \%$ of all cellranger singlet cells as 'real' single cells. Therefore, these cells were ascertained as singlets. To recover the cell assignment to the remaining 9.4\% cellranger singlets, predicted as doublets by Demuxlet, we utilised gene expression matrix to model cell doublets, using a simulation-based approach [45]. For each cell that was identified as a singlet by both Demuxlet and the doublet expression simulation, it was assigned to a donor based on the highest likelihood probability from Demuxlet. Doublets identified by Demuxlet or the doublet expression simulation were removed before downstream processing and analysis. 


\section{Normalisation}

Normalisation was conducted at four levels: between samples within a pool, between pools, between cells and between clusters. The between-pool normalisation followed the subsampling strategy in the cellranger pipeline, where the reads, genes and cells were randomly subsampled following subsampling rates determined by the total read per sample and binomial distribution [46]. Four pools were randomly multiplexed into one sequencing lane. For cellto-cell normalisation, a cell-pooling strategy was applied to circumvent the zero-inflation issue, as described by Lun et al. [47]. Between-pool normalisation followed Combat parametric empirical Bayesian strategy. To select the normalisation strategy, we compared results from using Combat, RUV and SCRAN methods by using $k$-BET batch-effect scores [18]. We found that a combination of SCRAN normalisation followed by Combat was superior in reducing batch effects compared to other methods, consistent with the results reported by Buttner and colleagues [18]. Prior to eQTL analysis, the mean expression of each gene per individual per cell subpopulation was computed, quantile normalised and z-transformed for eQTL mapping.

\section{Imputation and quality control of genotype data}

The 79 cell lines were genotyped by Infinium HumanCore-24 v1.1 BeadChip assay (Illumina). GenomeStudioTM V2.0 (Illumina) was used for SNP genotype calling of the BeadChip data (total 306,670 SNPs for one assay). The full genotype report files were reformatted into Plink map, fam and lgen files and were then converted into variant calling format (vcf) using custom shell scripts and Plink2 [48]. Plink2-converted files contained predicted reference and alternative alleles with no information for homozygous genotypes, which were fixed using the GenomeStudio report file and a custom script. For each sorted, indexed vcf file (separated by chromosomes), a strand fixing step was performed using bcf fixref function [49]. Prior to imputation, Eagle V.2.3.5 was used for haplotype phasing the strand-fixed genotype vcf files [50]. The phased data were imputed based on the 1000 genome phase 3 reference panel (2535 samples) using the minimac3 program in the Michigan Imputation server [51].

\section{Cell type classification and annotation}

We combined all cells from the fibroblasts and iPSC pools separately. We normalised and clustered the cells in these two datasets to ensure that the clustering was not affected by pool-specific data processing. We performed clustering using the SCORE method to identify subpopulations of cells [52]. Clustree [53] was used to display the cluster stability at different resolutions (Additional file 1: Figure S18). To visualise cell distributions, we used non-linear Uniform Manifold Approximation and Projection (UMAP) dimensionality reduction [54]. Cyclone [55] was used to estimate cell cycle stages of each cell. Pseudo-trajectory analysis was carried out with slingshot [56] using the UMAP cell projections.

We used gene regulatory network analysis independently on the two datasets-fibroblasts and iPSCs-to identify unique regulatory networks in each cell type with the pySCENIC method (v.0.10.3) using default parameters [57, 58]. The method involves three steps: (1) modules of genes that are co-expressed with transcription factors are identified from the correlation matrix using GRNboost algorithm [57]. (2) Then those modules were 
pruned by RcisTarget [58], a transcription factor motif enrichment analysis to identify cisregulatory motifs around the putative target genes. Only genes that contain the binding motif for each respective transcription factor are retained in the module. The gene sets in these modules-known as regulons-consist of each candidate transcription factor and their target genes. (3) Lastly, the 'activity' of each regulon is measured in each cell using the AUCell package [58] where the area under the recovery curve (AUC) measures the activity for the regulon in that cell by calculating whether a subset of the input gene set is enriched in the expressed genes. For each regulon and cell, the AUC values were calculated using AUCell_calcAUC function, where AUC values represent the fraction of genes within the top-ranking transcription factor that were defined in each cluster. We visualised the AUCell scores generated from SCENIC with ComplexHeatmap [59].

The UMAP projected 59 cells from the fibroblast $\mathrm{RXRB}^{+}$cell type in the upper right corner of the UMAP. To be sure these were not outlier cells, we tested for differentially expressed genes between the 'central cells' and the 'far cells' of this $\mathrm{RXRB}^{+}$cell type but only found four genes that were differentially expressed using a Wilcox test (Additional file 1: Figure S19). SingleR [19] was used to map single cell transcriptomes against 713 reference transcriptomes.

\section{eQTL association analysis}

To study specific regulation effects of genomic variance to gene expression, we performed statistical analysis of the association between genotypes of single nucleotide polymorphisms and single cell gene expression for 79 fibroblast cell lines and 31 iPSC cell lines generated from the same individuals. We filtered for common SNPs (minor allele frequency $>0.05$ ) that were within $\pm 1 \mathrm{Mb}$ of an expressed gene (detected in $>1 \%$ of the cells), resulting in 5,368,223 SNPs and 9796 genes for the fibroblasts, and 4,508, 778 SNPs and 10,899 genes for the iPSCs. SNP genotypes were recoded as 0,1 or 2 copies of the reference allele.

\section{Model choice}

To determine the best choice of model, we tested multiple models for detecting eQTLs with the SNPs and genes located on chromosome 21. In each case, we used a linear regression to detect eQTLs but compared models with different covariates and PEER factors, and with different normalisation of the input gene expression levels [60]. In total, we tested the following linear models:

1. Gene expression calculated as the average of across cells, per cell type, per individual. Then quantile normalised and $z$-transformed without any additional covariates

2. Gene expression calculated as the average of across cells, per cell type, per individual. Then quantile normalised and $z$-transformed with one PEER factor as a covariate

3. Gene expression calculated as the average of across cells, per cell type, per individual. Then quantile normalised and $z$-transformed with ten PEER factors as covariates

4. Using all cells for a given cell type, gene expression was quantile normalised and $z$ transformed, and a random effect to account for the non-independence of the dependent variable (multiple cells from the same individual) 
5. Using all cells for a given cell type with a random effect to account for the nonindependence of the dependent variable (multiple cells from the same individual)

The numbers of eQTLs detected on chromosome 21 for each of these models indicated that the first three models that used the average expression per gene for each cell type per individual resulted in the identification of the largest number of eQTLs (Additional file 11: Table S10). Therefore, we further interrogated the number of PEER factors to include in the model. The final model that we selected for genome-wide eQTL detection in each cell type used the average expression level of the cells per individual for that cell type and 1 PEER factor (Additional file 1: Figure S20). PEER factors were calculated using genes that passed QC filtering (9796 genes in fibroblast cell types and 10,899 genes for iPSC cell types).

eQTL mapping was performed for each subpopulation identified by the clustering analysis. Therefore, for each subpopulation, the average expression for a given individual in that cell subpopulatipon was used to detect cis-eQTLs. Cis-eQTLs (SNP $\leq 1 \mathrm{Mb}$ ) were detected using a linear model implemented in the MatrixEQTL R software with study-wide FDR lower than 10\% using the Benjamini-Hochberg procedure [61].

\section{Differential expression}

We used edgeR $[62,63]$ to identify differentially expressed genes between each cell type compared with the other cell types combined (i.e. each fibroblast type compared to the other five fibroblast types and each iPSC type compared to the other three iPSC types). Differentially expressed genes were detected using the gene-wise negative binomial generalised model with a quasi-likelihood test. Detection rate and pool batches were included as covariates following the recommendations of Soneson and Robinson [31]. Heatmaps and upset plots were generated using ComplexHeatmap [59] in R. Heatmaps were created with scaled, normalised data.

\section{Independent eQTL analysis}

Given an eGene that was significant in a pair of cell types ( $a$ and $b$ ), the top eSNPs from each cell type $\left(S_{a}\right.$ and $\left.S_{b}\right)$ were tested for independence with relation to eGene expression. Accordingly, the top eSNP $\left(S_{b}\right)$ in cell type $b$ was regressed from the linear model for the association of the top eSNP, $S_{a}$, for cell type $a$ with gene expression of the eGene $\left(G_{a}\right)$ in cell type $a$.

$$
G_{a} \sim \beta_{0}+\beta_{1} S_{a}+\beta_{2} S_{b}+P E E R_{1}+\varepsilon
$$

eSNPs were deemed independent if the association between $S_{a}$ and $G_{a}$ was significant following regression of $S_{b}$ in the linear model. This model works well for these data since all the scRNA-seq data for fibroblast and iPSC cells was generated, processed and underwent quality control assessment together.

\section{Interaction $\mathrm{eQTL}$ analysis}

Given an eGene that was significant in at least two cell types, the eSNP with the largest difference between their beta allelic effects between any two clusters was used to test for cell type interaction. Two models were fit for gene expression $G$, with SNP $S$ and cell type $C$. The first model (1) was a normal linear model and the second model (2) 
included an interaction term. An interaction was considered significant if an anova comparing the two models was significant.

1) $G \sim \beta_{0}+\beta_{1} S+\beta_{2} C+P E E R_{1}+\beta$

2) $G \sim \beta_{0}+\beta_{1} S+\beta_{2} C+\beta_{3} S C+P E E R_{1}+\beta$

eGene correlation

The expression of eGenes that were unique to a given cell type were correlated with their expression in the other cell types using a Pearson correlation test.

\section{Test statistic correlation}

The significant eQTL test statistics from each cell subtype was compared with test statistic for the same eGene-eSNP pair in all other cell types to test whether one contributing factor for the highly cell type-specific eQTL detection could be due to lack of power.

\section{eGene enrichment}

eGenes from a specific cell type were tested for enrichment in the other cell types. eGenes were ranked based on the lowest $p$ value for each eGene. An expected distribution of mean rank scores was generated from 10,000 permutations of randomly selected genes (selecting the same number of genes as eGenes). The mean rank of the eGenes in the testing cell types was then tested for significance with a $t$ test.

\section{GTEx comparison}

Gene Tissue Expression (GTEx) [22] database version seven results were downloaded on 6 July 2019. The cultured fibroblast cell eQTL were compared with the fibroblast cell type eQTL results to identify common and unique results.

\section{iPSC reprogramming comparison}

Genes differentially expressed during iPSC reprogramming from fibroblasts were obtained from Liu et al. [32]. Gene IDs were used to identify reprogramming genes that were also eQTLs. Differentially methylated regions (DMRs) between fibroblasts and iPSCs were obtained from Lister et al. [33] and were checked for overlapping eSNPs. DMRs between iPSCs and ESCs were obtained from Polo et al. [34] and were checked for eSNPs within $100 \mathrm{bp}$ of the differentially methylated base pair.

\section{Figure preparation and creation}

Plots panels were prepared in R with ggplot [64], Gviz [65], ComplexHeatmap [59] and LDlinkR [66]. They were arranged and edited with BioRender.com.

\section{Supplementary Information}

The online version contains supplementary material available at https://doi.org/10.1186/s13059-021-02293-3. 


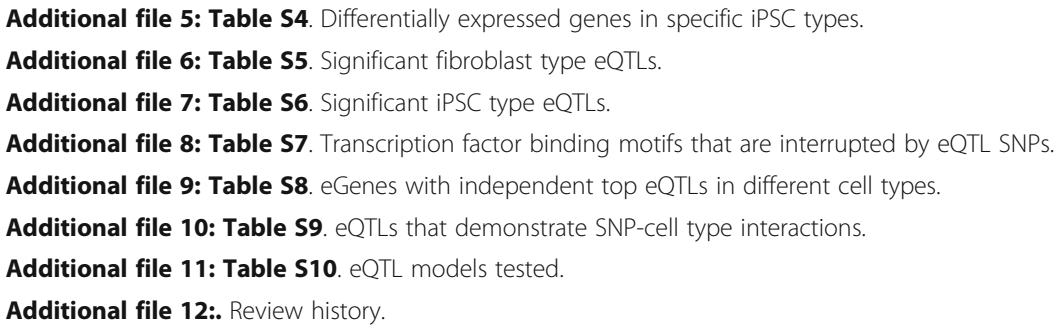

We are grateful to the participants who provided samples for this analysis. Thanks also to Antonio García for support with figures.

Review history

The review history is available as Additional file 12.

Peer review information

Barbara Cheifet was the primary editor of this article and managed its editorial process and peer review in collaboration with the rest of the editorial team.

\section{Authors' contributions}

Study conception: JEP, AWH, AP, NJP

Data analysis: DRN, QN, YW, AS, SWL

Sample collection: AP, AWH, MSD, HHL, DEC

Tissue culture and data production: MSD, HSC, GEL, DH

Manuscript writing: JEP, DRN, QN, AP, NJP, AWH

The authors read and approved the final manuscript.

\section{Funding}

This work was supported by grants from the National Health and Medical Research Council (NHMRC) project grant (APP1143163) and Australian Research Council discovery project (DP180101405). JEP is supported by an NHMRC Investigator grant (APP1175781). AWH is supported by an NHMRC Senior Practitioner Fellowship (APP1195444). AP is supported by an Australian Research Council Future Fellowship (FT140100047) and an NHMRC Senior Research Fellowship (APP1154389). NJP is supported by a Fellowship from the Australian Heart Foundation (101889). MD was supported by an International Postgraduate Research Scholarship \& Research Training Program Scholarship. This work is also supported by a special initiative from the Australian Research Council (SR1101002), the Joan and Peter Clemenger Foundation, the Ophthalmic Research Institute of Australia, Stem Cells Australia - the Australian Research Council Special Research Initiative in Stem Cell Science, the University of Melbourne and Operational Infrastructure Support from the Victorian Government.

\section{Availability of data and materials}

The scRNA-seq data for all 79 fibroblast cell lines and 31 iPSC cell lines are available from ArrayExpress (Accession Number: E-MTAB-10060) [67].

\section{Ethics approval and consent to participate}

Experimental work was approved by the Human Research Ethics committees of the Royal Victorian Eye and Ear Hospital (11/1031), University of Melbourne (1545394) and University of Tasmania (H0014124) in accordance with the requirements of the National Health \& Medical Research Council of Australia (NHMRC) and conformed with the Declaration of Helsinki [37]. All patients gave written informed consent to collect and use their biological data for this research.

\section{Competing interests}

The authors declare no competing interests.

\section{Author details}

${ }^{1}$ Garvan-Weizmann Centre for Cellular Genomics, Garvan Institute of Medical Research, Darlinghurst, Sydney, Australia. ${ }^{2}$ Institute for Molecular Bioscience, University of Queensland, Brisbane, Australia. ${ }^{3}$ Centre for Eye Research Australia, Royal Victorian Eye and Ear Hospital, Melbourne, Australia. ${ }^{4}$ Department of Surgery, The University of Melbourne, Melbourne, Australia. ${ }^{5}$ Department of Anatomy and Physiology, The University of Melbourne, Melbourne, Australia. ${ }^{6}$ Wicking Dementia Research and Education Centre, University of Tasmania, Hobart, Australia. ${ }^{7}$ School of Medicine, Menzies Institute for Medical Research, University of Tasmania, Hobart, Australia. ${ }^{8}$ UNSW Cellular Genomics Futures Institute, School of Medical Sciences, University of New South Wales, Sydney, Australia.

Received: 8 June 2020 Accepted: 10 February 2021

Published online: 05 March 2021

\section{References}

1. Rockman MV, Kruglyak L. Genetics of global gene expression. Nat Rev Genet. 2006:862-72. https://doi.org/10.1038/nrg1964. 
2. Nica AC, Dermitzakis ET. Expression quantitative trait loci: present and future. Philosophical Transactions of the Royal Society B: Biological Sciences. 2013. p. 20120362. https://doi.org/10.1098/rstb.2012.0362

3. Hormozdiari F, Gazal S, van de Geijn B, Finucane HK, CJ-T J, Loh P-R, et al. Leveraging molecular quantitative trait loci to understand the genetic architecture of diseases and complex traits. Nat Genet. 2018:50:1041-7.

4. Gamazon ER, Segrè AV, van de Bunt M, Wen X, Xi HS, Hormozdiari F, et al. Using an atlas of gene regulation across 44 human tissues to inform complex disease- and trait-associated variation. Nat Genet. 2018;50:956-67.

5. Ding J, Gudjonsson JE, Liang L, Stuart PE, Li Y, Chen W, et al. Gene expression in skin and lymphoblastoid cells: refined statistical method reveals extensive overlap in cis-eQTL signals. Am J Hum Genet. 2010;87:779-89.

6. Pickrell JK, Marioni JC, Pai AA, Degner JF, Engelhardt BE, Nkadori E, et al. Understanding mechanisms underlying human gene expression variation with RNA sequencing. Nature. 2010;464:768-72.

7. Fu J, Wolfs MGM, Deelen P, Westra H-J, Fehrmann RSN, Te Meerman GJ, et al. Unraveling the regulatory mechanisms underlying tissue-dependent genetic variation of gene expression. Plos Genet. 2012;8:e1002431.

8. Consortium G, GTEx Consortium. Genetic effects on gene expression across human tissues. Nature. 2017:204-13. https:// doi.org/10.1038/nature24277.

9. Nguyen QH, Lukowski SW, Chiu HS, Senabouth A, Bruxner TJC, Christ AN, et al. Single-cell RNA-seq of human induced pluripotent stem cells reveals cellular heterogeneity and cell state transitions between subpopulations. Genome Res. 2018;28:1053-66.

10. Vieira Braga FA, Kar G, Berg M, Carpaij OA, Polanski K, Simon LM, et al. A cellular census of human lungs identifies novel cell states in health and in asthma. Nat Med. 2019;25:1153-63.

11. Park J, Shrestha R, Qiu C, Kondo A, Huang S, Werth M, et al. Single-cell transcriptomics of the mouse kidney reveals potential cellular targets of kidney disease. Science. 2018;360:758-63.

12. Aizarani N, Saviano A, Sagar ML, Durand S, Herman JS, et al. A human liver cell atlas reveals heterogeneity and epithelial progenitors. Nature. 2019;572:199-204.

13. Cuomo ASE, Seaton DD, McCarthy DJ, Martinez I, Bonder MJ, Garcia-Bernardo J, et al. Single-cell RNA-sequencing of differentiating iPS cells reveals dynamic genetic effects on gene expression. https://doi.org/10.1101/630996

14. van der Wijst MGP, Brugge H, de Vries DH, Deelen P, Swertz MA, LifeLines Cohort Study, et al. Single-cell RNA sequencing identifies celltype-specific cis-eQTLs and co-expression QTLs. Nat Genet. 2018;50:493-7.

15. Wills QF, Livak KJ, Tipping AJ, Enver T, Goldson AJ, Sexton DW, et al. Single-cell gene expression analysis reveals genetic associations masked in whole-tissue experiments. Nat Biotechnol. 2013;31:748-52.

16. Kang HM, Subramaniam M, Targ S, Nguyen M, Maliskova L, McCarthy E, et al. Multiplexed droplet single-cell RNAsequencing using natural genetic variation. Nat Biotechnol. 2018;36:89-94.

17. Sarkar AK, Tung P-Y, Blischak JD, Burnett JE, Li YI, Stephens M, et al. Discovery and characterization of variance QTLs in human induced pluripotent stem cells. PLoS Genet. 2019;15:e1008045.

18. Büttner M, Miao Z, Alexander Wolf F, Teichmann SA, Theis FJ. Assessment of batch-correction methods for scRNA-seq data with a new test metric. bioRxiv. 2017. p. 200345. Available from: https://www.biorxiv.org/content/10.1101/20034 5V2.abstract. [cited 2019 Aug 10]

19. Aran D, Looney AP, Liu L, Wu E, Fong V, Hsu A, et al. Reference-based analysis of lung single-cell sequencing reveals a transitional profibrotic macrophage. Nat Immunol. 2019;20:163-72.

20. Mabbott NA, Baillie JK, Brown H, Freeman TC, Hume DA. An expression atlas of human primary cells: inference of gene function from coexpression networks. BMC Genomics. 2013;14:632.

21. Denu RA, Nemcek S, Bloom DD, Goodrich AD, Kim J, Mosher DF, et al. Fibroblasts and mesenchymal stromal/stem cells are phenotypically indistinguishable. Acta Haematol. 2016;136:85-97.

22. Carithers LJ, Ardlie K, Barcus M, Branton PA, Britton A, Buia SA, et al. A novel approach to high-quality postmortem tissue procurement: the GTEx project. Biopreserv Biobank. 2015;13:311-9.

23. Jansen R, Hottenga J-J, Nivard MG, Abdellaoui A, Laport B, de Geus EJ, et al. Conditional eQTL analysis reveals allelic heterogeneity of gene expression. Hum Mol Genet. 2017;26:1444-51.

24. Has C. The "Kelch" surprise: KLHL24, a new player in the pathogenesis of skin fragility. J Invest Dermatol. 2017;137:1211-2.

25. Gupta VA, Beggs AH. Kelch proteins: emerging roles in skeletal muscle development and diseases. Skelet Muscle. 2014;4:11.

26. Salomonis N, Dexheimer PJ, Omberg L, Schroll R, Bush S, Huo J, et al. Integrated genomic analysis of diverse induced pluripotent stem cells from the Progenitor Cell Biology Consortium. Stem Cell Reports. 2016;7:110-25.

27. Shi C, Li H, Couturier JP, Yang K, Guo X, He D, et al. Allele specific expression of MICA variants in human fibroblasts suggests a pathogenic mechanism. Open Rheumatol J. 2015;9:60-4.

28. Pashos EE, Park Y, Wang X, Raghavan A, Yang W, Abbey D, et al. Large, diverse population cohorts of hiPSCs and derived hepatocyte-like cells reveal functional genetic variation at blood lipid-associated loci. Cell Stem Cell. 2017;20: 558-70.e10.

29. Park N, Yoo JC, Ryu J, Hong S-G, Hwang EM, Park J-Y. Copine1 enhances neuronal differentiation of the hippocampal progenitor HiB5 cells. Mol Cells. 2012;34:549-54.

30. Kim TH, Sung S-E, Yoo JC, Park J-Y, Yi G-S, Heo JY, et al. Copine1 regulates neural stem cell functions during brain development. Biochem Biophys Res Commun. 2018:168-73. https://doi.org/10.1016/j.bbrc.2017.10.167.

31. Soneson C, Robinson MD. Bias, robustness and scalability in single-cell differential expression analysis. Nat Methods. 2018;15:255-61.

32. Liu X, Ouyang JF, Rossello FJ, Tan JP, Davidson KC, Valdes DS, et al. Reprogramming roadmap reveals route to human induced trophoblast stem cells. Nature. 2020;586:101-7.

33. Lister R, Pelizzola M, Kida YS, Hawkins RD, Nery JR, Hon G, et al. Hotspots of aberrant epigenomic reprogramming in human induced pluripotent stem cells. Nature. 2011;471:68-73.

34. Polo JM, Liu S, Figueroa ME, Kulalert W, Eminli S, Tan KY, et al. Cell type of origin influences the molecular and functional properties of mouse induced pluripotent stem cells. Nat Biotechnol. 2010;28:848-55.

35. Guo C, Kong W, Kamimoto K, Rivera-Gonzalez GC, Yang X, Kirita Y, et al. CellTag Indexing: genetic barcode-based sample multiplexing for single-cell genomics. Genome Biol. 2019;20:90.

36. Biddy BA, Kong W, Kamimoto K, Guo C, Waye SE, Sun T, et al. Single-cell mapping of lineage and identity in direct reprogramming. Nature. 2018;564:219-24. 
37. McCaughey T, Liang HH, Chen C, Fenwick E, Rees G, Wong RCB, et al. An interactive multimedia approach to improving informed consent for induced pluripotent stem cell research. Cell Stem Cell. 2016;18:307-8.

38. Okita K, Matsumura Y, Sato Y, Okada A, Morizane A, Okamoto S, et al. A more efficient method to generate integrationfree human iPS cells. Nature Methods. 2011:409-12. https://doi.org/10.1038/nmeth.1591.

39. Crombie DE, Daniszewski M, Liang HH, Kulkarni T, Li F, Lidgerwood GE, et al. Development of a modular automated system for maintenance and differentiation of adherent human pluripotent stem cells. SLAS Discov. 2017;22:1016-25

40. Daniszewski M, Nguyen Q, Chy HS, Singh V, Crombie DE, Kulkarni T, et al. Single-cell profiling identifies key pathways expressed by iPSCs cultured in different commercial media. iscience. 2018;7:30-9.

41. Colella S, Yau C, Taylor JM, Mirza G, Butler H, Clouston P, et al. QuantiSNP: an objective Bayes hidden-Markov model to detect and accurately map copy number variation using SNP genotyping data. Nucleic Acids Research. 2007:2013-25. https://doi.org/10.1093/nar/gkm076.

42. Wang K, Li M, Hadley D, Liu R, Glessner J, Grant SFA, et al. PennCNV: an integrated hidden Markov model designed for high-resolution copy number variation detection in whole-genome SNP genotyping data. Genome Res. 2007:1665-74. https://doi.org/10.1101/gr.6861907.

43. 10x Genomics. Available from: http://10xgenomics.com/. [cited 2020 Sep 1]

44. Dobin A, Davis CA, Schlesinger F, Drenkow J, Zaleski C, Jha S, et al. STAR: ultrafast universal RNA-seq aligner. Bioinformatics. 2013;29:15-21.

45. Dahlin JS, Hamey FK, Pijuan-Sala B, Shepherd M, Lau WWY, Nestorowa S, et al. A single-cell hematopoietic landscape resolves 8 lineage trajectories and defects in Kit mutant mice. Blood. 2018;131:e1-11.

46. Zheng GXY, Terry JM, Belgrader P, Ryvkin P, Bent ZW, Wilson R, et al. Massively parallel digital transcriptional profiling of single cells. Nat Commun. 2017;8:14049.

47. Lun ATL, Bach K, Marioni JC. Pooling across cells to normalize single-cell RNA sequencing data with many zero counts. Genome Biol. 2016;17:75.

48. Chang CC, Chow CC, Tellier LC, Vattikuti S, Purcell SM, Lee JJ. Second-generation PLINK: rising to the challenge of larger and richer datasets. Gigascience. 2015:4:7.

49. Li H. A statistical framework for SNP calling, mutation discovery, association mapping and population genetical parameter estimation from sequencing data. Bioinformatics. 2011;27:2987-93.

50. Loh P-R, Palamara PF, Price AL. Fast and accurate long-range phasing in a UK Biobank cohort. Nat Genet. 2016; 48:811-6.

51. Das S, Forer L, Schönherr S, Sidore C, Locke AE, Kwong A, et al. Next-generation genotype imputation service and methods. Nat Genet. 2016;48:1284-7.

52. Satija R, Farrell JA, Gennert D, Schier AF, Regev A. Spatial reconstruction of single-cell gene expression data. Nat Biotechnol. 2015;33:495-502.

53. Zappia L, Oshlack A. Clustering trees: a visualisation for evaluating clusterings at multiple resolutions. https://doi.org/1 $0.1101 / 274035$

54. Becht E, McInnes L, Healy J, Dutertre C-A, Kwok IWH, Ng LG, et al. Dimensionality reduction for visualizing single-cell data using UMAP. Nat Biotechnol. 2018. https://doi.org/10.1038/nbt.4314.

55. Scialdone A, Natarajan KN, Saraiva LR, Proserpio V, Teichmann SA, Stegle O, et al. Computational assignment of cellcycle stage from single-cell transcriptome data. Methods. 2015;85:54-61.

56. Street K, Risso D, Fletcher RB, Das D, Ngai J, Yosef N, et al. Slingshot: cell lineage and pseudotime inference for singlecell transcriptomics. BMC Genomics. 2018;19:477.

57. Van de Sande B, Flerin C, Davie K, De Waegeneer M, Hulselmans G, Aibar S, et al. A scalable SCENIC workflow for singlecell gene regulatory network analysis. Nat Protoc. 2020;15:2247-76.

58. Aibar S, González-Blas CB, Moerman T, Huynh-Thu VA, Imrichova H, Hulselmans G, et al. SCENIC: single-cell regulatory network inference and clustering. Nat Methods. 2017;14:1083-6.

59. Gu Z, Eils R, Schlesner M. Complex heatmaps reveal patterns and correlations in multidimensional genomic data. Bioinformatics. 2016;32:2847-9.

60. Stegle O, Parts L, Piipari M, Winn J, Durbin R. Using probabilistic estimation of expression residuals (PEER) to obtain increased power and interpretability of gene expression analyses. Nat Protoc. 2012;7:500-7.

61. Shabalin AA. Matrix eQTL: ultra fast eQTL analysis via large matrix operations. Bioinformatics. 2012;28: $1353-8$.

62. Robinson MD, McCarthy DJ, Smyth GK. edgeR: a Bioconductor package for differential expression analysis of digital gene expression data. Bioinformatics. 2010;26:139-40.

63. McCarthy DJ, Chen Y, Smyth GK. Differential expression analysis of multifactor RNA-Seq experiments with respect to biological variation. Nucleic Acids Res. 2012;40:4288-97.

64. Wickham H. ggplot2: elegant graphics for data analysis. 2nd ed. New York City: Springer International Publishing; 2016.

65. Genomics S. Methods and protocols. Anticancer Res. 2016;36:3224.

66. Myers TA, Chanock SJ, LDlinkR MMJ. An R package for rapidly calculating linkage disequilibrium statistics in diverse populations. Front Genet. 2020;11:157.

67. Neavin D, Nguyen Q, Daniszewski MS, Liang HH, Sheng H, Chui HS, Wee YK, Senabouth A, Lukowski SW, Crombie DE, Lidgerwood GE, Hernandez D, Vickers JC, Cook AL, Palpant NJ, Pebay A, Hewitt AW, Powell JF. Single cell $\mathrm{eQTL}$ analysis identifies cell type-specific genetic control of gene expression in fibroblasts and reprogrammed induced pluripotent stem cells. Datasets. ArrayExpress. 2021. https://www.ebi.ac.uk/arrayexpress/ experiments/E-MTAB-10060/. Accessed 23 Feb 2021.

\section{Publisher's Note}

Springer Nature remains neutral with regard to jurisdictional claims in published maps and institutional affiliations. 